\title{
Estrategias de marketing para microempresas comercializadoras de productos autoadhesivos, caso pyme Maplas
}

DOI: https://doi.org/10.33262/ap.v3i4.111

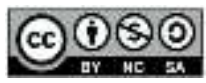

Marketing strategies for micro-companies that sell self-adhesive products, case of MAPLAS

Viviana Melissa Cuadra Jama. ${ }^{1} \&$ Jessenia Maribel Granja Viteri. ${ }^{2}$

\begin{abstract}
Introduction: Marketing constitutes a fundamental tool in the development of any type of company where the dissemination and commercialization of products and/or services in the market is sought; with the appropriate application of actions that lead to the fulfillment of set objectives. Marketing strategies seek to frame the right path for the fulfillment of objectives defined in a marketing plan that allows taking advantage of the opportunities that the market offers. Objective: To determine the use of marketing strategies for micro-companies commercializing self-adhesive products, specifically in the case of MAPLAS, a small and medium-sized company. Methodology: The research corresponds to a non-experimental field design with a population of 103 SMEs registered in the SRI registry in 2020; the application of surveys to a sample determined by stratified sampling with the application of the formula constitutes 81 observed data that responded to a 20-item questionnaire. Results: Among the results, $67.2 \%$ make monthly purchases of self-adhesive products; people prefer to make their purchases in stores where there is good customer service and a variety of products; users of self-adhesive products prefer products with the main characteristic of the degree of adhesion. Conclusion: It was concluded that the diffusion of products in the SME Maplas should be oriented to satisfy the needs of customers who are looking for quality products.
\end{abstract}

\footnotetext{
1 Instituto Superior Tecnológico Vicente León, Latacunga, Ecuador, v.cuadra@istvicenteleon.edu.ec, https://orcid.org/0000-0001-7235-7402

2 Instituto Superior Tecnológico Vicente León, Latacunga, Ecuador, j.granja@istvicenteleon.edu.ec, https://orcid.org/0000-0002-8160-2075
} 
Keywords: quality, commercialization, strategies, marketing, media.

\section{Resumen}

Introducción: El marketing constituye una herramienta fundamental en el desarrollo de cualquier tipo de empresa en donde se busca la difusión y comercialización de productos y/o servicios en el mercado; con la aplicación adecuada de acciones que conlleven al cumplimiento de objetivos planteados. Las estrategias de marketing buscan enmarcar el camino correcto para el cumplimiento de objetivos definidos en un plan de marketing que permitan aprovechar las oportunidades que el mercado ofrece. Objetivo: Determinar el uso de las estrategias de marketing para microempresas comercializadoras de productos autoadhesivos, específicamente al caso pyme Maplas. Metodología: La investigación corresponde a un diseño no experimental de campo con una población de 103 pymes que constan en el registro del SRI año 2020; la aplicación de encuestas a una muestra determinada por muestreo estratificado con aplicación de la fórmula constituye 81 datos observados que dieron respuesta a un cuestionario de 20 ítems. Resultados: Entre los resultados el 67,2\% realizan compras mensuales de productos autoadhesivos, las personas prefieren realizar sus compras en los locales en donde otorguen una buena atención al cliente y exista variedad de productos; los usuarios de autoadhesivos prefieren los productos con característica principal el grado de adherencia. Conclusión: Se concluyó que la difusión de productos en la pyme Maplas se debe orientar a la satisfacción de necesidades de los clientes quienes buscan artículos de calidad.

Palabras claves: calidad, comercialización, estrategias, marketing, medios de comunicación.

\section{Introducción}

La implementación de estrategias de marketing para microempresas comercializadoras de productos autoadhesivos basada en la pyme MAPLAS, es una investigación significativa dentro del sector comercial, ya que permite a la pyme especializarse en un nicho de mercado específico y ganar una imagen en la medida que satisfaga las necesidades de los clientes potenciales, diseñando una mezcla de marketing integrado, conformado por factores que controla el producto, precio, plaza y promoción. Con estas actividades, la Pyme observa, analiza y se adapta a diseñar un plan de acción que consiga los objetivos deseados.

El Marketing es importante, puesto que aplica métodos, basados en el arte de atraer clientes potenciales, que se sientan satisfechos y lograr fidelizar a los mismos. En términos generales, el marketing es una estrategia que contempla los procedimientos de ventas y la relación entre la empresa y los clientes.

La comercialización de productos autoadhesivos se desarrolla desde que el producto sale del establecimiento del fabricante hasta que llega al consumidor final, es así que, al 
mercadear el producto, impulsa la toma de decisiones de compra entre los clientes y es necesario utilizar métodos o estrategias para atraer a los consumidores.

El proceso actual de la globalización está ocasionando que las empresas, independientemente del tamaño y los mercados que abastezcan, se integren a la competencia internacional. Encontramos que los principales factores que determinan la competitividad en las empresas de Latinoamérica son: la innovación, la productividad, la calidad, el desarrollo del capital humano, la inversión en investigación y desarrollo y finalmente la gestión financiera (Ycaza \& Sánchez-Riofrío, 2016).

Las pymes en Latinoamérica con el entorno comercial globalizado, la puesta en marcha de áreas de libre comercio, dieron origen a un conjunto de transformaciones en las decisiones sobre la forma de crear y establecer estrategias de marketing, para enfrentarse a estos retos y no correr el riesgo de desparecer.

En la región latinoamericana es necesario implementar programas de cooperación y alianzas estratégicas, con el fin de conformar sistemas comerciales que respondan a las condiciones actuales y futuras (Díaz, 2014).

La internacionalización representa en el actual contexto económico una estrategia fundamental en la gestión empresarial. La evolución de los sistemas de comunicación, la reducción de costos de transporte, la importancia de alianzas empresariales y la reducción de barreras de comunicación, son las estrategias principales en el mundo en el que estamos viviendo.

En Ecuador, en concordancia a la propuesta de cambio de la matriz productiva, se hace necesario potenciar el enfoque en un proceso de aprendizaje que lleve a la identificación, construcción y aprovechamiento de las oportunidades de negocio que brindan los mercados globalizados, con una visión planificadora a largo plazo que reactive la productividad nacional, la competitividad de las empresas y el desarrollo de clúster. Al realizar un análisis crítico en la pyme MAPLAS, se evidencia que los bajos niveles de venta son causados por algunos factores que se detallan a continuación dando causas y efectos que no permiten un desempeño apropiado de la pyme.

\section{Metodología}

La presente investigación constituye un estudio del nivel descriptivo ya que se analiza las diferentes circunstancias que influyen en una problemática, se definen las características y se limita el espacio de toma de información a una determinada población.

En función a esta premisa la presente investigación está sustentada en un análisis cualitativo sobre la implementación de estrategias de marketing para las microempresas dedicadas a la comercialización de productos autoadhesivos, sustentados en la investigación desarrollada en Maplas; un estudio cualitativo busca interpretar los criterios de las personas quienes fueron objeto de un proceso de investigación que considera un método inductivo - deductivo ya que se parte desde el planteamiento de un problema para determinar sus causas y efectos. 
Para la recolección de información se aplicó una encuesta por medio de un cuestionario, instrumento que permitió recopilar información en base a veinte interrogantes planteadas conforme las variables de estudio.

Ante esto, Arias (2012) explica que la metodología del proyecto incluye "el tipo de investigación, las técnicas e instrumentos que serán utilizados para llevar a cabo la investigación. Es el cómo se realizará el estudio para responder al problema planteado" (p. 110).

La metodología incluye una serie de aspectos, que permitirán al investigador indagar y proponer soluciones a la problemática planteada. Según Hernández et al. (2007), definen al marco metodológico cómo “...el apartado del trabajo y dará el giro a la investigación, es donde se expone la manera como se va a realizar el estudio, los pasos para realizarlo, su método." (p. 150).

Arias (2012), estipula que la técnica es "el procedimiento o forma particular de obtener datos o información" (p.67.). En respuesta al planteamiento, se indica que se aplicará la encuesta online como técnica para obtener información, para tal término señala que es "una técnica que pretende obtener información que suministra un grupo o muestra de sujetos acerca de sí mismos, o en relación con un tema y particular" (p.72.).

Para esta premisa, Sabino (1992) explica el instrumento de recolección de datos como: “...cualquier recurso que se vale el investigador para acercarse a los fenómenos y a extraer de ellos información.” (p. 143.)

El instrumento a utilizar para la técnica de la encuesta escrita, será el cuestionario que Arias (2012) lo define como "la modalidad de encuesta que se realiza de forma escrita mediante un instrumento o formato en papel contentivo de una serie de preguntas...". (p.74).

\section{Marco Teórico}

\section{Marketing}

El marketing tiene dos fases, la primera de ser filosofía, perspectiva u orientación administrativa; con fines de satisfacción del cliente. La segunda fase, mecánica; que consiste en el marketing, como una serie de actividades, que se utilizan para implementar esta filosofía (Lamb \& McDaniel, 2011).

Según Walker \& Stanton (2007) el marketing es un conjunto de actividades de negocios, ideal para la planeación de productos satisfactores de necesidades, provistos de un precio, promoción y distribución a los mercados, a fin de lograr los objetivos de la organización.

El marketing, se caracteriza por la realización de actividades; que tienen por objetivo alcanzar las metas de una organización, se anticipa a los requerimientos del consumidor y canaliza un flujo de mercancías, es decir enrolando necesidades y servicios, que el productor presta al consumidor o cliente. Según McCarthy \& Perreault (2000) plantea 
que "la mercadotecnia es un trabajo de procesos para producir, informar, proporcionar e intercambiar ofertas; que tienen valor para los consumidores, clientes, socios y para la sociedad en general".

Para esta investigación el Marketing como aproximación teórica según el trabajo expuesto por los diferentes referentes teóricos mencionados, representa un conjunto actividades científicas, que basan su trabajo en el arte de explorar, crear y entregar valor para satisfacer necesidades de un mercado objetivo con lucro. Esta concepción identifica necesidades y deseos no realizados. Define, mide y cuantifica el tamaño del mercado identificado y el lucro potencial, debido a que es una disciplina que transforma la realidad para generar y obtener valor, anticipándose a las necesidades de los clientes potenciales. Esta aproximación teórica es aplicada a las Pymes, desde un análisis de estrategias en personas, precio, productos y promociones.

Las estrategias de mercadeo, pueden lograrse a través del departamento de mercadeo, como cambios en la publicidad, énfasis en las promociones, canales de distribución, diseño del producto o estrategias de precio (Gómez \& Villalba, 2018).

Según Magaña \& de Mercadotecnia (2016), las estrategias de marketing deben de contar con funciones especializadas en la cual se desarrollan técnicas y métodos; como el uso de redes sociales para promocionar los productos y captar clientes potenciales.

\section{Estado del Arte de marketing}

El marketing es un conjunto de acciones y procesos; al momento de crear un producto, "comunicar, entregar valor a los clientes", cuyo objetivo es beneficiar a la organización, en la satisfacción de sus clientes. Durante décadas el marketing como filosofía ha evolucionado constantemente, un ejemplo de ello son las diversas orientaciones bajo las cuales ha operado y posteriormente transformado:

a) Orientación hacia la producción, según Lamb \& McDaniel (2011) es una filosofía centrada para el interior de las organizaciones, relacionada a la capacidad productiva y creativa de las empresas; y no así los deseos o necesidades de los consumidores, ¡ésta orientación es conocida como el Marketing del "field of dream" o "el campo de los sueños" y su compromiso básico es "Build it and they will come!!!" "constrúyelo y ellos vendrán!!!”. Evidentemente la producción es incompleta, porque no considera si los bienes y servicios que la empresa produce con mayor eficiencia; si no satisfacer correctamente las necesidades del mercado, un ejemplo de ello es la frase de Henry Ford, fundador de la compañía Ford Motor Company "Puedo producir un automóvil de cualquier color siempre y cuando sea negro".

b) Orientación Hacia las Ventas, según Lamb \& McDaniel (2011), se fundamenta en la filosofía de la premisa, donde las personas compran más productos y servicios si se utilizan técnicas de ventas y que las grandes ventas rinden utilidades. Bajo esta filosofía el marketing, representa vender cosas y cobrar dinero, considerando la calidad de los productos o la relación costo beneficio que éstos logran generar. De 
tal modo, la filosofía hacia la producción, se considera incompleta puesto que tampoco tiene en cuenta al consumidor, solo apunta a la formación de una fuerza de ventas numerosas y muy agresiva.

c) Orientación hacia el mercado, según Lamb \& McDaniel (2011), hace mención a una filosofía sencilla y atractiva que afirma, los aspectos social y económico, en su razón fundamental de la existencia de una organización, consiste en deleitar los deseos y necesidades de los clientes, a la par se alcanza los objetivos: "Lo que la empresa vende no es de importancia, lo primordial, es lo que los clientes creen estar comprando, por lo tanto, es lo que define el negocio".

d) Orientación social, según Lamb \& McDaniel (2011), menciona, que la filosofía de la orientación social y destinado al mercado son las de mayor impacto y trascendencia en la actualidad. Dicha orientación social gira en torno a las organizaciones, no solo para satisfacer los deseos y necesidades de los clientes; sino también para velar por los intereses de los individuos y de la sociedad. Ofertando productos menos tóxicos, más duraderos, más amables con el medio ambiente, reutilizables o hechos de material reciclable.

En sus inicios el marketing trataba de analizar los problemas derivados del binomio producción-producto, y trataba de buscar la eficiencia en la distribución. A partir de aquel tiempo, los distintos autores han propuesto diferentes etapas por donde consideran que evolucionó el concepto de marketing.

Precedentes (anterior al siglo XX): incluye los antecedentes de la revolución industrial, la era de la producción, la división del trabajo y el desarrollo del comercio identificaron ocho etapas en el desarrollo del marketing, resumidas de la siguiente forma:

a. Aparición (1900-1920), es cuando aparecen en Estados Unidos los primeros institutos de investigación de mercados y también cuando se identifican funciones empresariales distintas de la producción. Se comienza a utilizar el término marketing, cuando se emplea por primera vez en la Universidad de Wisconsin, además da origen a las primeras asociaciones profesionales del marketing.

b. Conceptual (1921-1945), establecimiento de una diferenciación de productos según la política comercial de las empresas y se crea la American Marketing Asociation.

c. Desarrollo (1946-1960), el marketing se introduce en el campo de los bienes industriales. Jerome McCarthy en 1960, desarrolla su propio paradigma de las 4Ps de marketing, y da lugar a la evolución del concepto desde la transferencia exclusiva de bienes a la satisfacción de necesidades y deseos.

d. Expansión (1960-1970), Kotler y Levy en 1969 publican en el Journal of Marketing un artículo que marcará el futuro del marketing al ampliar su campo de actuación más allá del ámbito empresarial. 
e. Desarrollo conceptual (1970-1980), inician a introducirse los conceptos y técnicas del marketing en el sector social.

f. Consolidación (1980-1990), los problemas, resultados y las teorías de marketing se clasifican en función de tres categorías dicotómicas: (1) sector con ánimo de lucro y el sin ánimo de lucro, (2) nivel de agregación, tanto macro y micro y (3) análisis positiva y normativa.

g. Revisión y tendencias (a partir de 1990), nueva orientación al consumidor, a la competencia, a la coordinación interfuncional dentro de la empresa o institución y al marketing de redes.

\section{Estrategias de marketing y mezcla de marketing}

Kotler \& Armstrong (2013) mencionan, las empresas saben que no pueden atender de manera rentable a todos los clientes de un determinado mercado (al menos no a todos de la misma manera). Existen demasiados tipos distintos de consumidores con demasiadas clases de necesidades. La mayoría de las empresas están en posición de atender a algunos segmentos mejor que a otros. Así, cada empresa debe dividir el mercado entero, elegir los mejores segmentos y diseñar estrategias para atender de manera rentable a los segmentos que eligió. Este proceso implica la segmentación de mercado, la selección de mercado meta, la diferenciación y el posicionamiento.

La mezcla de marketing es el conjunto de herramientas de marketing que la empresa combina para producir la respuesta que desea en el mercado meta. La mezcla de marketing consiste en todo lo que la empresa puede hacer para influir en la demanda de su producto. Las múltiples posibilidades pueden ser agrupadas en cuatro grupos de variables: las cuatro Ps.

Producto significa la combinación de bienes y servicios que la empresa ofrece al mercado meta.

Precio es la cantidad de dinero que los clientes deben pagar para obtener el producto.

Plaza incluye actividades de la empresa encaminadas a que el producto esté disponible para

los clientes meta.

Promoción se refiere a las actividades que comunican los méritos del producto y persuaden a los clientes meta a comprarlo.

Un programa de marketing eficaz combina cada elemento de la mezcla de marketing en un programa de marketing integrado diseñado para alcanzar los objetivos de marketing de la empresa entregando valor a los clientes. La mezcla de marketing constituye el kit de herramientas tácticas de la empresa para establecer un fuerte posicionamiento en los mercados meta. 


\section{Comercialización}

Esta definición representa un hecho sublime en el marco investigativo planteado, la cual se define como la venta de un producto. Se utiliza también este término para referirse a la última fase o lanzamiento de un nuevo producto. En general, la comercialización se define como un conjunto de actividades comerciales llevadas a cabo por la empresa, la cual comprende diversas etapas: análisis del mercado, planificación del producto, fijación del precio, selección de canales y distribución física del producto, promoción del producto y organización y control de los planes de actuación comercial.

\section{Tipos de comercialización}

\section{- Distribuidor}

Tipo de intermediario muy similar al mayorista, pero les diferencia algo clave. Mientras que los mayoristas disponen de una amplia variedad de productos de la competencia; los distribuidores sólo llevan líneas de productos complementarios y tienen una relación muy estrecha con sus proveedores y clientes. Además, los distribuidores son propietarios de los productos y los almacenan ellos mismos hasta que finalmente los venden.

\section{- Mayorista}

Figura que vende todo tipo y gran variedad de productos, almacenándolos y vendiéndolos, ya sea a otras empresas o a consumidores finales. Aunque, normalmente, este tipo de intermediario vende los productos a otros intermediarios, generalmente a minoristas.

\section{- Minorista}

Otro de los intermediarios en la comercialización de productos son los minoristas. Éstos se dedican a la venta de productos al por menor o al detalle. Es el intermediario que vende directamente al consumidor los productos que previamente ha comprado.

\section{- Corredor}

También llamado bróker, es un tipo de intermediario en la comercialización de productos que sirve de enlace entre compradores y vendedores; pero con la gran diferencia de que nunca toma posesión física de ningún producto (al contrario que el minorista, por ejemplo). La labor del corredor es la de reunir a compradores y vendedores y, a cambio, se lleva una comisión por el éxito de la venta.

\section{- Agentes comerciales}

Persona independiente cuyo principal objetivo es el de actuar como vendedor principal de un producto, siendo su representante frente a los usuarios. El agente comercial toma posesión del producto, aunque en realidad no lo posee (pues no lo compra como hace un minorista). Por sus servicios, obtienen unas comisiones. 
Los tipos de intermediarios en la comercialización de productos son varios. Cada empresa, en función del territorio en el que actúe, el volumen y tamaño de sus pedidos, ubicación y otros muchos elementos y necesidades, decidirá cuál es la figura con la que más le interesa colaborar.

\section{Resultados}

Del grupo de personas encuestadas el $67.2 \%$ realiza compras de PRODUCTOS AUTOADESIVOS con frecuencia mensual el $17.2 \%$ lo realiza semanal, mientras que un $12.1 \%$ opta por realizar compras con una frecuencia quincenal, el porcentaje restante representa la compra diaria, lo que indica que el mercado para vender el producto es amplio.

Del grupo de personas encuestadas se evidencia, mayor aceptación en cuanto al producto en la marca ARCLAD (empresa enfocada en el desarrollo y la fabricación de materiales autoadhesivos), realidad que indica que el mercado para vender el producto es amplio.

En este punto se puede evidenciar un aporte fundamental para la investigación sobre los productos autoadhesivos, de lo cual se expone ciertas ventajas en cada una de las categorías expuestas las mismas que permiten proponer varios criterios esenciales para el desarrollo e implementación de estrategias para la comercialización de productos; se hace énfasis en la preferencia de los consumidores a los productos elaborados a base de vinilos ya que se considera un material de calidad en la elaboración de autoadhesivos.

Los consumidores de estos productos referencian la calidad de los materiales y la relación costo beneficio considerando como factores fundamentales al momento de realizar sus compras. Otro factor importante constituye los criterios de variedad y promoción porque el cliente puede escoger entre las múltiples opciones que el vendedor le otorgue.

Las empresas además de ofrecer productos de calidad deben brindar una buena atención a sus clientes antes, durante y después de la compra, considerando todas aquellas acciones que beneficien la venta y comercialización de productos. La comunicación directa para proporcionar suficiente información, la retroalimentación en la comunicación permitirá satisfacer las dudas, inquietudes o sugerencias que pueden tener los clientes.

La publicidad y los medios de comunicación para las pymes constituye un elemento de gran importancia ya que son fuentes de difusión que permiten dar a conocer las empresas que existen y los productos que ofertan. Al ser un análisis de un caso particular se determina que los mejores medios de difusión constituyen los medios tradicionales como lo es la televisión, radio e incursionar con la publicidad por redes sociales. Los medios tradicionales están dirigidos a personas adultas quienes se han resistido a la innovación tecnológica mientras que las redes sociales constituyen una herramienta básica e imprescindible hoy en día, ya que todas las personas al menos manejan una red social.

Las campañas publicitarias por medio de promociones constituyen otra manera de captar clientes, el uso de esta estrategia de marketing tiene como finalidad el informar, persuadir 
y recordar al público objetivo sobre los productos que se comercializan en las empresas. Al utilizar esta herramienta los productos que son difíciles de comercializar se vuelven llamativos y se pueden vender de manera rápida, tomando en consideración que las promociones se estipulan en un tiempo determinado. La estrategia de promoción tiene como finalidad el influir en la actitud y comportamiento de los consumidores.

El mensaje de la publicidad constituye el objeto de la comunicación. Este está definido como la información o enunciado verbal que el emisor envía al receptor a través de un canal de comunicación o medio de comunicación determinado. La difusión de un mensaje publicitario debe constituirse como un elemento de comunicación estructurado tomando en consideración las emociones, los factores culturales, sociales e informativos que se pueden divulgar con facilidad.

Toda transacción comercial tiene como elemento particular una forma de pago, que en la actualidad se realiza por diferentes medios ya sea por tarjetas de crédito, débito o transacciones bancarias en el caso particular de las pymes deben actualizar su sistema de pago para que no se restrinja a los clientes en el uso de efectivo, ya que puede constituirse un limitante al momento de la compra.

Una estrategia de marketing que se puede aplicar para el desarrollo de las pymes es la cooperación corporativa que tiene como objetivo el impulsar el desarrollo organizacional, el trabajo colaborativo es muy beneficioso para mejorar el rendimiento y los productos de una empresa, este tipo de alianza permite una sinergia de esfuerzos direccionados a un mismo fin.

Esta investigación ha permitido obtener datos y direccionarlos hacia nuevas estrategias de marketing. De este modo, el propósito central es posicionar los productos autoadhesivos marca Arclad, tanto en el mercado como en la mente del consumidor; con la implementación de nuevas estrategias se logrará la diferenciación de la pyme MAPLAS, en relación a las características del resto de empresas en similares condiciones.

La posibilidad de que el negocio sea encontrado por clientes potenciales aumenta cuando el catálogo es publicado en Internet; ya que permite que éste sea visto por una gran cantidad de personas que están interesadas en los bienes y/o servicios que la empresa pone a disposición de los consumidores.

Los catálogos online permiten clasificar y jerarquizar la información de una manera más efectiva; ya que la navegación debe guiar al usuario a conseguir lo que busca por múltiples maneras de una forma rápida y eficiente.

Los catálogos virtuales eliminan los costos de papel, de impresión y de distribución; a diferencia de los catálogos tradicionales. Así como también, disminuyen el gasto en el diseño, la composición, la producción y el envío. De igual manera, la facilidad para hacerle cambios ahorra recursos; ya que no es necesario volverlos a imprimir para realizar las actualizaciones del catálogo. 
Un catálogo que sea distribuido o expuesto en un medio tan universal como el Internet, genera mayor presencia de la marca y de los productos en el mercado. Al igual que, facilita el proceso de dar a conocer nuevos productos o de mantener en la mente del consumidor los ya existentes. Así como también, Al ser una representación gráfica de la empresa y de los productos que vende; se refuerza la imagen que los clientes tienen de la organización.

\section{Conclusiones}

El estudio realizado tuvo como parte esencial la microempresa Maplas de la ciudad de Latacunga, investigación que podría ser referente en el estudio de diferentes pymes que se dedican a la comercialización de productos autoadhesivos.

- El planteamiento de la filosofía empresarial y el manejo adecuado de las estrategias de marketing permiten posicionar a las pymes en el mercado de productos autoadhesivos, mediante la fidelización de clientes, alianzas estratégicas con proveedores y clientes potenciales.

- Se debe mantener un catálogo de productos actualizado para la exhibición en donde se identifique la marca, el mismo debe ser atrayente, con fotos de buena calidad de tal modo que los clientes puedan apreciar con detalle los atributos de los productos, además debe contar con un sistema de codificación para organizar la mercadería en favor tanto del cliente como del personal de la tienda, quienes pueden brindar la información específica de un producto. También el catálogo debe presentar variedad, de modo que los clientes puedan escoger los productos de su preferencia de acuerdo a su gusto.

- Se debe conservar la calidad de los productos, aunque este aspecto obliga a que los precios de venta sean más altos, considerando la calidad como un elemento visible en las tiendas virtuales con productos similares a los que se comercializan en las pymes pese a que el nivel de precios es muy diferencial; el estilo y la calidad apuntan a consumidores exigentes.

- La implementación de un catálogo online constituye una ventaja competitiva en razón de la digitalización de los procesos en la comercialización de productos, ya que constituye una herramienta de fácil acceso para cualquier persona; la incorporación de estas herramientas dinamiza la actividad comercial.

\section{Referencias Bibliográficas}

Arias, F. G. (2012). El proyecto de investigación. Introducción a la metodología científica. 6ta. Fidias G. Arias Odón.

Díaz, J. A. (2014). Comercialización de los productos y servicios de la ciencia: retos y perspectivas. Revista Cubana de Ciencia Agrícola, 48(1), 21-24. 
Gómez, L. D., \& Villalba, M. R., (2018). Estrategias de publicidad y el posicionamiento de mercado de las ferreterías de la ciudad de Latacunga. (Trabajo de investigación). Repositorio de la Universidad Técnica de Ambato. Recuperado de https://repositorio.uta.edu.ec/jspui/handle/123456789/27984

Hernández Fernández, C., Ristol Pont, J., Estivill, E., Batista Miranda, J. E., \& Aramburu, L. (2007). Importancia de la nocturia y su impacto en la calidad del sueño y en la calidad de vida en el paciente con hiperplasia benigna de próstata. Actas Urológicas Españolas, 31(3), 262-269.

Kotler, P., \& Armstrong, G. (2013). Fundamentos de marketing. Pearson Educación

Lamb, C. W., Hair, J. F., \& McDaniel, C. (2011). Essentials of marketing. Cengage Learning.

Magaña Marquez, J. E., \& de Mercadotecnia, S.(2016). Implementación de marketing en el mundo digital, estrategias de las pequeñas y medianas empresas.

McCarthy, E. J., \& Perreault, W. D. (2000). Marketing un enfoque global. McGraw-Hill.

Sabino, C. (1992). El conocimiento científico. C. Sabino, El proceso de investigación.

Walker, S. E., Etzel, M., \& Stanton, W. (2007). Fundamentos de marketing. Editorial McGraw Hill.

Ycaza, D. E. M., \& Sánchez-Riofrío, A. M. (2016). Factores de competitividad orientados a la pequeña y mediana empresa (PYME) en Latinoamérica: revisión de la literatura. Revista San Gregorio, 2(15), 104-111.

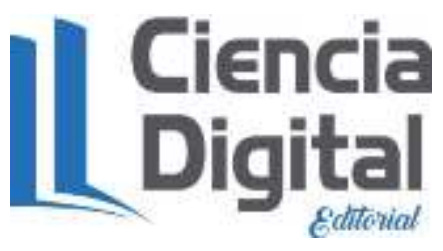




\section{PARA CITAR EL ARTÍCULO INDEXADO.}

Cuadra Jama, V. M., \& Granja Viteri, J. M. (2021). Estrategias de marketing para microempresas comercializadoras de productos autoadhesivos, caso pyme Maplas. AlfaPublicaciones, 3(4), 55-67. https://doi.org/10.33262/ap.v3i4.111

\section{Liencia}

El artículo que se publica es de exclusiva responsabilidad de los autores y no necesariamente reflejan el pensamiento de la Revista Alfa Publicaciones.

El artículo queda en propiedad de la revista y, por tanto, su publicación parcial y/o total en otro medio tiene que ser autorizado por el director de la Revista Alfa Publicaciones.
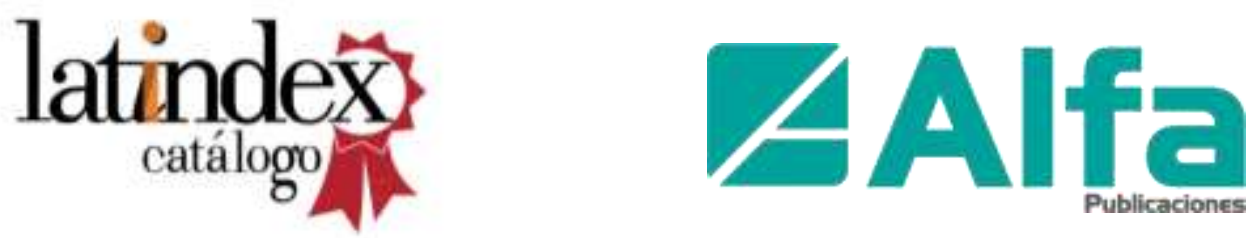